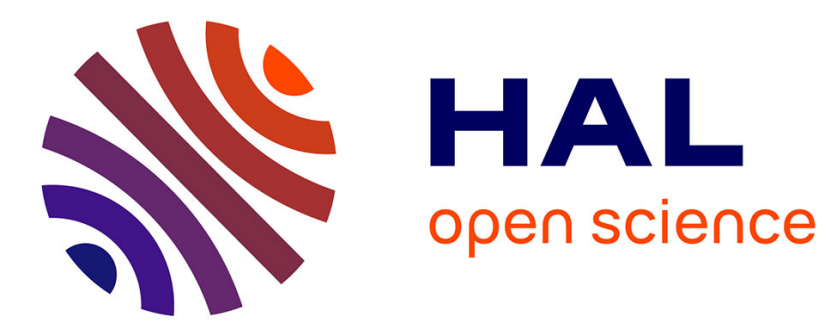

\title{
Local interactions and the global organization of a two-phase flow in a branching tree
}

\author{
Yu Song, Paul Manneville, Charles N. Baroud
}

\section{To cite this version:}

Yu Song, Paul Manneville, Charles N. Baroud. Local interactions and the global organization of a two-phase flow in a branching tree. Physical Review Letters, 2010, 105 (13), pp.134501. 10.1103/physrevlett.105.134501 . hal-01020669

\section{HAL Id: hal-01020669 \\ https://hal-polytechnique.archives-ouvertes.fr/hal-01020669}

Submitted on 9 Jul 2014

HAL is a multi-disciplinary open access archive for the deposit and dissemination of scientific research documents, whether they are published or not. The documents may come from teaching and research institutions in France or abroad, or from public or private research centers.
L'archive ouverte pluridisciplinaire HAL, est destinée au dépôt et à la diffusion de documents scientifiques de niveau recherche, publiés ou non, émanant des établissements d'enseignement et de recherche français ou étrangers, des laboratoires publics ou privés. 


\title{
Local Interactions and the Global Organization of a Two-Phase Flow in a Branching Tree
}

\author{
Yu Song, Paul Manneville, and Charles N. Baroud* \\ LadHyX and Department of Mechanics, Ecole Polytechnique, CNRS UMR 7646, 91128 Palaiseau Cedex, France
}

(Received 3 May 2010; published 23 September 2010)

\begin{abstract}
The transport of liquid plugs in a microfluidic branching tree is studied experimentally. The global flow pattern can be either symmetric or asymmetric, with daughter plugs dividing in synchrony or asynchrony as a function of the driving flow rate and the network geometry. For trees with narrowing channels, the plugs always reach the exits even at low flow rates. In contrast, only one path is opened in networks with widening channels when the flow rate is low. This behavior is explained by a comparison of the pressure drop necessary to drive viscocapillary motion of plugs in straight channels with the nonlinear pressure variations as a plug passes a bifurcation. A model is built, which predicts that only narrowing networks can be fully filled, while widening networks can never be fully invaded by a two-phase flow.
\end{abstract}

DOI: 10.1103/PhysRevLett.105.134501

Large-scale rearrangements in connected networks have attracted much attention in recent years, mostly in theoretical studies of networks as models for social interactions, disease propagation, and electrical and fluid transport $[1,2]$. In the case of fluid transport in interconnected geometries, complex dynamics can arise when the local pressure-flow-rate relations are nonlinear, for example, when the flow takes place in compliant conduits [3,4] or in the presence of immiscible interfaces [5]. In such situations, the local flow-rate fluctuations lead to instantaneous, global reequilibration of the pressure, thus producing long-range couplings in the flow. This can play a dominant role for flows in porous or biological media, where the evolution of multiphase flows occurs through a competition between viscous and capillary effects, which are both influenced by the local details of the geometry.

Microfluidic devices have been proposed for modeling such flows since they offer fine control over the geometry, in addition to providing detailed optical access to the fluid behavior (see, e.g., [6]). However, most previous studies have considered the statistics of transport on a global scale, usually by coarse-graining the local dynamics. This has limited the ability to build networks with prescribed characteristics, such as the ones studied theoretically, due to a lack of local constitutive relations that can be generalized. Here, we fill this gap by presenting a deterministic study of the advance of a two-phase flow in a complex network.

We are particularly motivated by two-phase flows that take place in the pulmonary airways and therefore focus on binary branching trees. The air passages in the lung can be occluded by liquid plugs either in pathological situations, such as Respiratory Distress Syndrome, or as a method for drug delivery in which medication is administered as a liquid bolus into the airways. Indeed, such treatment is already commonplace among premature infants, who suffer from a deficit of surfactant in the lung and whose survival depends on succeeding to flow some surfactant into the distal branches of the airways [7]. While models of
PACS numbers: 47.55.-t, 47.61.Jd, 47.63.-b, 47.56.+r

liquid transport in bifurcating airways have already been explored in experiments, both in vitro $[8,9]$ and ex vivo [10], in addition to numerical models $[11,12]$, there remains a large gap between the microscopic understanding of the motion of a single plug and the organization of the flow on a large scale in a network of channels.

We use microfluidic techniques to build two binary branching networks with different recursion rules and observe the flow of a single liquid plug that is pushed into each. Networks, such as the one shown in Fig. 1, are made of polydimethylsiloxane (PDMS) using soft lithography techniques [13]. Perfluorodecalin is used to make the liquid plugs. It has viscosity $\eta=5 \times 10^{-3} \mathrm{~Pa}$ s and surface tension $\gamma=20 \times 10^{-3} \mathrm{~N} / \mathrm{m}$ and also provides good wetting conditions on the PDMS surfaces. The channel height at all

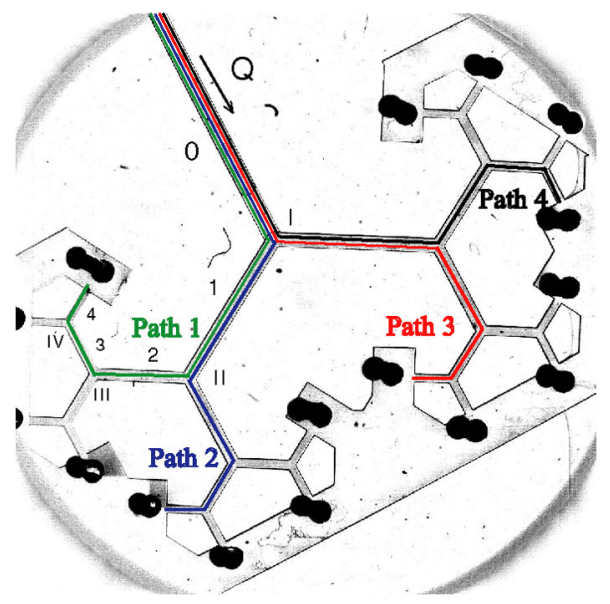

FIG. 1 (color online). Image of a narrowing network with 5 generations. Generation (bifurcation) numbers are labeled with Arabic (Roman) numerals. A constant flow rate is applied through the network inlet, at generation 0. Sixteen holes are made at the exits to fix the boundary condition at atmospheric pressure. The four paths along which measurements are made are marked. 
branches is $b=50 \mu \mathrm{m}$, while its width $w_{i}$ varies by a constant factor $\rho: w_{i+1}=\rho w_{i}$, where the subscript denotes the generation number. Two networks are studied: a narrowing network with $\rho=0.83$ (referred to later as the $\mathcal{N}$ network) and a widening network with $\rho=1.2$ ( $\mathcal{W}$ network). The channel widths range from 342 to $720 \mu \mathrm{m}$ in both cases.

A typical experiment consists of creating a liquid plug at the root of the network and then pushing it at a constant flow rate $Q$ while recording its motion with a fast camera. At each bifurcation, the plug divides into two daughters of nearly equal size, and these daughters are transported in their respective branches. The positions of the daughter plugs are followed as they travel through four representative paths, as labeled in Fig. 1, and their traveled distances from the entrance are plotted in Figs. 2 and 3.

When pushed at a low flow rate of $Q=2 \mu \mathrm{l} / \mathrm{min}$ in the $\mathcal{N}$ network, the plug initially flows in generation 0 until it reaches the first bifurcation at which it divides into two daughters: The single solid black line in Fig. 2(a) thus leads to two lines (solid black and dashed blue). The dashed one then stops when it reaches bifurcation II, while the solid one passes and its daughters (solid black and dash-dotted red) advance to bifurcation III, where they now stop. At this point, the dashed plug divides through bifurcation II and catches up with the early ones. The difference in traveled distance between the dashed and solid lines, which had increased up to one generation, decreases again. When all of the daughters have arrived at bifurcation III, they divide and travel to the next bifurcation one by one.

The evolution for $Q=5 \mu \mathrm{l} / \mathrm{min}$ is similar except that the dashed plug divides at bifurcation II while the daughters of the solid are still moving in generation 2, corresponding

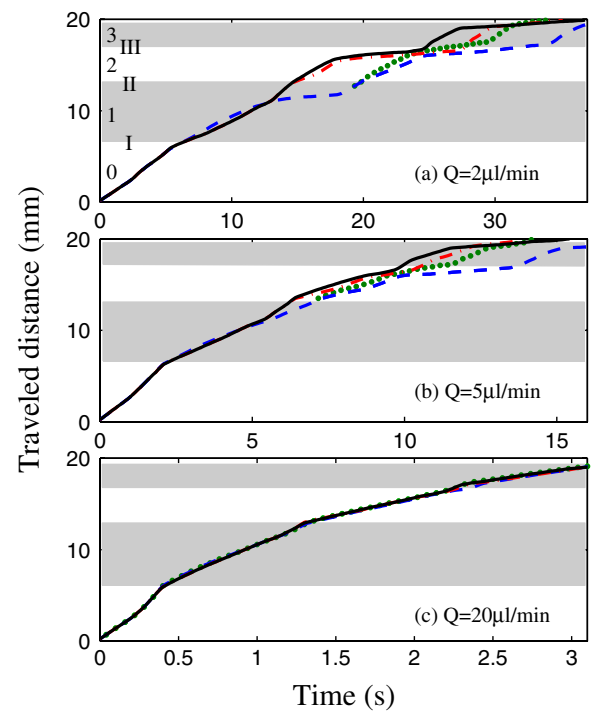

FIG. 2 (color online). Traveled distance along four paths as a function of time in the $\mathcal{N}$ network. Driving flow rates: (a) 2, (b) 5, and (c) $20 \mu \mathrm{l} / \mathrm{min}$. The shaded and nonshaded areas represent successive generations. to a period when flow exists in both branches. Three daughters also pass bifurcation III and travel through generation 3 simultaneously, followed by the dashed daughter that advances on its own. Finally, all the daughters advance simultaneously at high flow rates $(Q=20 \mu \mathrm{l} / \mathrm{min})$, and the distance plots are all superposed in this case. In all cases, the liquid penetrates everywhere in the $\mathcal{N}$ network regardless of the driving flow rate.

The flow patterns in the $\mathcal{W}$ network are shown in Fig. 3 . The initial behavior for $Q=2 \mu \mathrm{l} / \mathrm{min}$ is similar to that in the $\mathcal{N}$ network, but a difference arises at bifurcation II, where the dashed daughter of the initial plug is blocked and remains stationary until the end of the experiment. The other plug divides and its daughters reach bifurcation III, where only the dash-dotted red daughter continues, leaving the solid black one stationary. Since there is no flow in branches downstream of a bifurcation where a plug is blocked, the flux is limited to only a quarter of the network on the level of generation 3 when $Q=2 \mu \mathrm{l} / \mathrm{min}$. As for $Q=4 \mu \mathrm{l} / \mathrm{min}$, daughters of the initial plug pass bifurcation II successively, but only two daughters (solid and dash-dotted) pass through bifurcation III, while the other two get blocked. In the end, flow is set up in half of the network. When the flow is pushed at $Q=20 \mu \mathrm{l} / \mathrm{min}$, the daughters pass bifurcation II together but the crossings of bifurcation III are not simultaneous, resulting in asynchronous advance of the daughters afterward. Nevertheless, the whole network is penetrated at this high flow rate.

To understand the flow distribution, note that the presence of a liquid plug in the network modifies the dynamics in two ways. First, when the plug moves in a straight channel, it introduces a resistance to flow through its viscosity and surface tension. The relation between the viscocapillary pressure $P_{\mathrm{vc}}$ and the capillary number $\mathrm{Ca}=\eta U / \gamma$, for a plug of length $L$, can be written as [5]

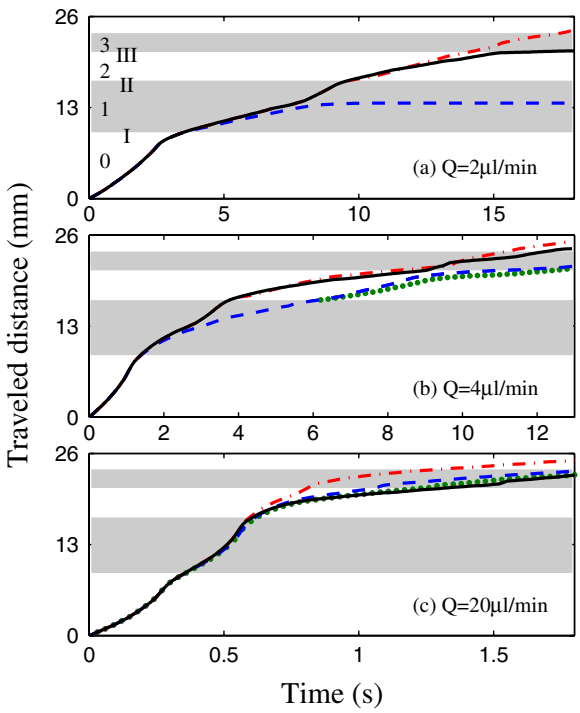

FIG. 3 (color online). Traveled distance in the $\mathcal{W}$ network. Driving flow rates: (a) 2, (b) 4, and (c) $20 \mu \mathrm{l} / \mathrm{min}$. 


$$
P_{\mathrm{vc}}=F(w) L \mathrm{Ca}+G(w) \mathrm{Ca}^{2 / 3},
$$

where $F(w)$ and $G(w)$ are functions of the channel width $w$. Since the plugs are driven at an imposed flow rate here, the value of $P_{\mathrm{vc}}$ can be obtained from Eq. (1) with the plug velocity calculated as $U=Q /(w b N)$, where $b$ is the channel height, $w$ is the channel width in the $i$ th generation, and $N$ is the number of moving plugs in that generation, ranging from 1 to $2^{i}$.

The second effect appears when the plug crosses a bifurcation, which we treat here as a quasistatic process unlike Ref. [5]. In this case, interface deformations imply position-dependent pressure variations, since the curvature of the front interface decreases and then increases again, as shown in Figs. 4(a) and 4(b). At a given plug position, there exists therefore a capillary pressure difference $P_{\text {cap }}$ which may resist the driving force:

$$
P_{\text {cap }}=\gamma / r_{r}-\gamma / r_{a},
$$

where $r_{r}$ and $r_{a}$ are the signed radii of curvature of the rear and front interfaces, respectively. The evolution of the interface shapes during this passage can be calculated from geometric constrains, by considering the angle of the bifurcation and the contact angle between the liquid and the PDMS, while imposing a constant curvature on the interface. As the plug advances, $P_{\text {cap }}$ increases before rapidly decreasing as it touches the opposite wall, as shown in Fig. 4(c).
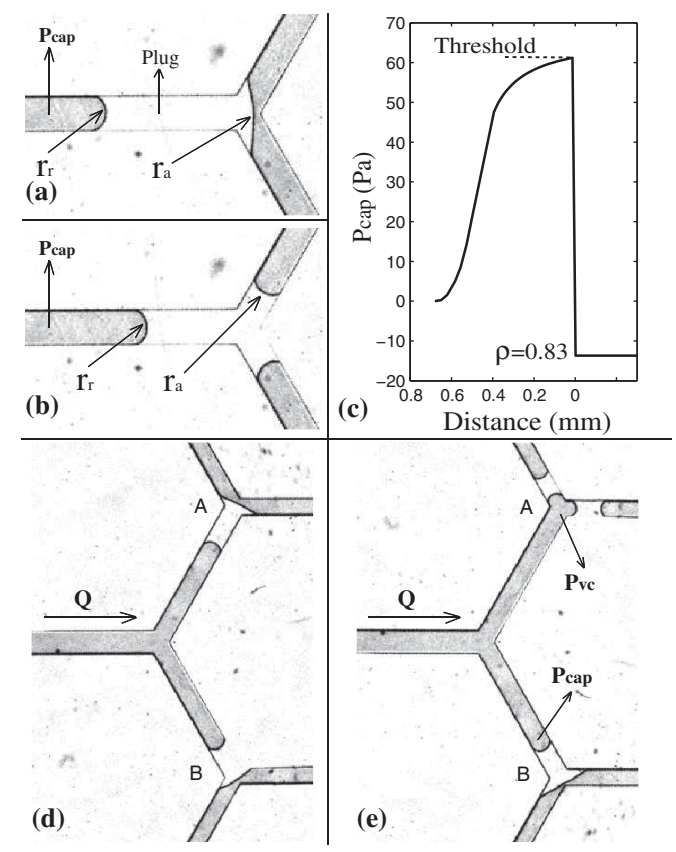

FIG. 4. Micrographs showing a liquid plug entering a bifurcation (a) and after touching the opposite wall (b). The radius of curvature at the front interface changes value and sign during this passage. (c) The variation in $P_{\text {cap }}$ versus the distance of the front interface from the facing tip. The calculation is performed by using Eq. (2) and for bifurcation II in the $\mathcal{N}$ network. (d), (e) Mesoscale building block for exploring long-range interactions in the tree.
This calculation shows the existence of a threshold pressure necessary to push the plug through a given bifurcation. It corresponds to the maximum value of $P_{\text {cap }}$. In the same way as for $w_{i}, P_{\text {thr }}$ varies with the generation number as $P_{\mathrm{thr}, i+1}=(1 / \rho) P_{\mathrm{thr}, i}$. This leads to $P_{\mathrm{thr}, i+1}>P_{\mathrm{thr}, i}$ in the $\mathcal{N}$ network, indicating that it is easier for the plugs located in bifurcation $i$ to pass than the plugs in bifurcation $i+1$. The distance between faster and slower daughters therefore cannot exceed one generation, in agreement with the experimental observations in Fig. 2. Conversely, in the $\mathcal{W}$ network, the distance between plugs can grow indefinitely since $P_{\mathrm{thr}, i}$ continuously decreases.

These nonlinear pressure variations in the bifurcation lead to long-range interactions across distinct subregions of the network when several plugs are present. A unit consisting of three connected bifurcations, as shown in Figs. 4(d) and 4(e), represents the basic building block to understand these interactions and explain the global behavior in the tree. The image in Fig. 4(d) is taken as the two daughters, $A$ and $B$, enter a bifurcation. Here, the front interfaces of the plugs are both deformed, and the common pressure $\Delta P$ driving the two plugs is given by $P_{\text {cap }}$. Slight variations in the geometry are always present in the experiments, leading to one of the plugs dividing before its sister [Fig. 4(e)]. Given that the flux in the system is imposed by the syringe pump, $\Delta P$ will adjust to satisfy the relation of Eq. (1), evaluated for the two daughters of $A$ moving at the velocity $U=Q / 2 w b$. The behavior of plug $B$ can then be deduced by comparing $P_{\mathrm{vc}}$ for the moving daughters of plug $A$ and $P_{\mathrm{thr}}$ for the bifurcation. If $P_{\mathrm{vc}}<P_{\mathrm{thr}}$, plug $B$ adjusts its position to equilibrate $P_{\text {cap }}=\Delta P$ and stays blocked. If $P_{\mathrm{vc}}>P_{\mathrm{thr}}$, plug $B$ passes the bifurcation, leading to a new value of $\Delta P$ that can be calculated by using Eq. (1) for four daughter plugs moving in the next generation.

This process has to be generalized to the case from 1 to $2^{i-1}$ plugs dividing at the $i$ th bifurcation, which gives a range of possible values for $P_{\mathrm{vc}}=\Delta P$ in the $i$ th generation, as shown by the shaded areas of Fig. 5. As more plugs pass the bifurcation they have entered, the number $N$ of moving plugs increases and $\Delta P$ decreases. As long as $\Delta P>P_{\text {thr }}$, the late plugs continue to divide and introduce more daughters, which lowers $\Delta P$ even more. When $\Delta P<P_{\text {thr }}$, some plugs in the $i$ th bifurcation remain blocked at a position where $P_{\text {cap }}=\Delta P$ is achieved. The relative values of $P_{\mathrm{vc}}$ and $P_{\mathrm{thr}}$ for each generation are given in Fig. 5 for the two networks. In that figure, the top line of each shaded area corresponds to one plug dividing, while the bottom line shows the pressure when all the plugs have divided.

The main difference between the two networks appears once an early plug that has divided reaches the next bifurcation. The threshold pressures associated with successive bifurcations, plotted by the $(+)$ symbols in Fig. 5, must be compared at this stage and the plug with the lower threshold will divide. In the $\mathcal{N}$ network, the upstream plugs always divide before plugs in the downstream 

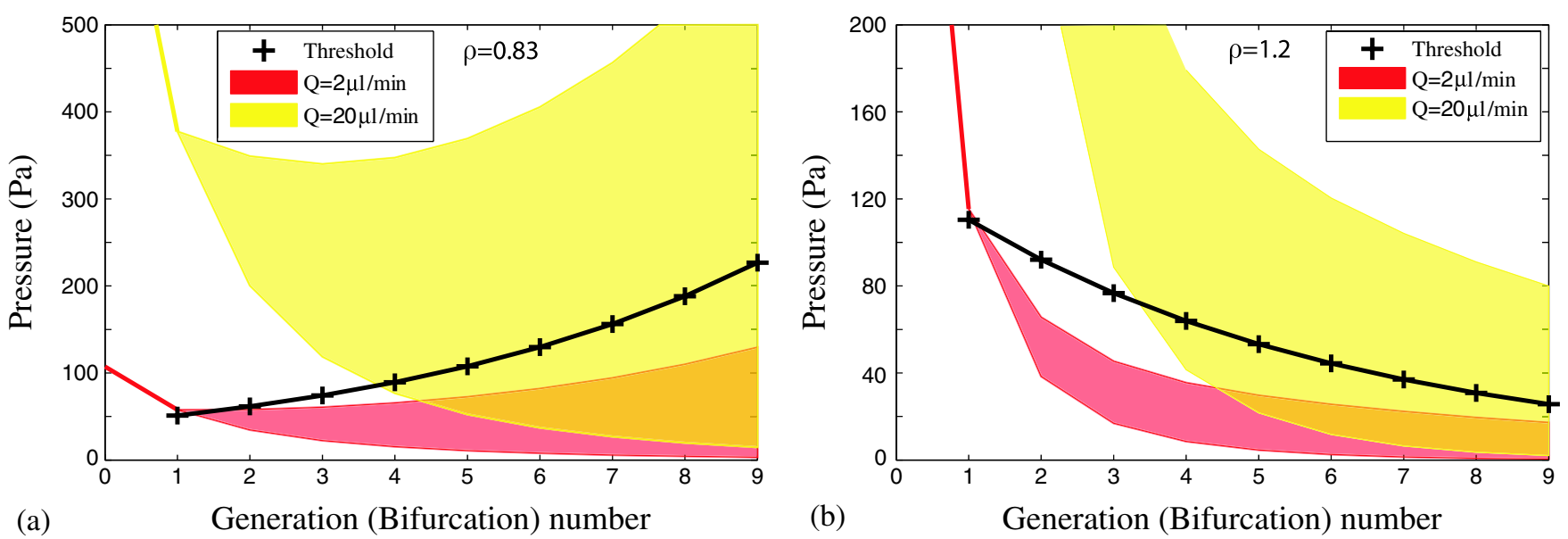

FIG. 5 (color online). Comparison of threshold pressures at every bifurcation to the pressures imposed in the straight channels by the driving flow rates $Q=2$ and $20 \mu \mathrm{l} / \mathrm{min}$. Labels on the $x$ axis denote the bifurcation number for the threshold and the generation number for the viscocapillary pressure. (a) $\mathcal{N}$ network and (b) $\mathcal{W}$ network.

bifurcation. In contrast, the downstream plug always divides first in the $\mathcal{W}$ network, and the upstream plug can no longer advance once it is stuck at the bifurcation.

The above argument thus shows that all the plugs can reach the exits in the $\mathcal{N}$ network regardless of driving flow rate since the early daughters wait for others at the downstream bifurcation. When $Q=2 \mu \mathrm{l} / \mathrm{min}$, we have $P_{\mathrm{vc}}<P_{\mathrm{thr}}$ and plugs advance one after another, in agreement with the experimental observation in Fig. 2(a). Since $P_{\mathrm{vc}}>P_{\mathrm{thr}}$ in the first four generations when $Q=$ $20 \mu \mathrm{l} / \mathrm{min}$, the plugs move together, as confirmed by the experiment in Fig. 2(c). In the region where $P_{\text {thr }}$ overlaps $P_{\mathrm{vc}}$, some of the daughters first advance simultaneously but will be caught up by the latecomers at a downstream bifurcation. In contrast, when $P_{\mathrm{vc}}<P_{\mathrm{thr}}$ in the $\mathcal{W}$ network, only one daughter can advance while all the others are blocked at different level bifurcations, in agreement with Fig. 3(a) for $Q=2 \mu \mathrm{l} / \mathrm{min}$. However, since $P_{\mathrm{vc}}>$ $P_{\text {thr }}$ for $Q=20 \mu \mathrm{l} / \mathrm{min}$, all the daughters are pushed to the exits. Given a network with more generations, we can predict that some plugs will stop before reaching the exits due to the strong decrease of $P_{\mathrm{vc}}$ with the generation number, as shown in Fig. 5(b).

In conclusion, we have identified a mesoscale element consisting of three adjacent bifurcations, which coarse grains the microscopic interactions and which can be used to build any network topology. The global transport in the network can now be inferred from an analysis of interactions at this mesoscale and between different mesoelements. In our fluid transport case, the evolution depends on the interplay between a dynamically determined pressure $\left(P_{\mathrm{vc}}\right)$ and a geometrically controlled threshold value $\left(P_{\text {thr }}\right)$, which is the source of the dominant nonlinearities. Generalizing these results to trains of multiple plugs is straightforward [14] and can lead to insights for many technological applications [15] or for flows in porous media [16], where viscocapillary balance is important.
More broadly, such threshold switching behavior is found in many networks beyond fluid mechanics [1,2], and the mesoscale approach can be adapted to the general case.

The authors acknowledge useful discussions with Michael Baudoin, James Grotberg, and David Grier.

*baroud@ladhyx.polytechnique.fr

[1] S. H. Strogatz, Nature (London) 410, 268 (2001).

[2] M.E. J. Newman, SIAM Rev. 45, 167 (2003).

[3] P. D. Howell, S. L. Waters, and J. B. Grotberg, J. Fluid Mech. 406, 309 (2000).

[4] A. L. Hazel and M. Heil, J. Fluid Mech. 478, 47 (2003).

[5] C.P. Ody, C. N. Baroud, and E. de Langre, J. Colloid Interface Sci. 308, 231 (2007).

[6] R. Lenormand, C. Zarcone, and A. Sarr, J. Fluid Mech. 135, 337 (1983); S. Krishnamurthy and Y. Peles, Phys. Fluids 19, 043302 (2007); V. Berejnov, N. Djilali, and D. Sinton, Lab Chip 8, 689 (2008).

[7] W. A. Engle and the Committee on Fetus \& Newborn, Pediatrics 121, 419 (2008).

[8] K. J. Cassidy, N. Gavriely, and J. B. Grotberg, J. Biomech. Eng. 123, 580 (2001).

[9] C. N. Baroud, S. Tsikata, and M. Heil, J. Fluid Mech. 546, 285 (2006).

[10] K. J. Cassidy et al., J. Appl. Physiol. 90, 1955 (2001).

[11] D. Halpern, O.E. Jensen, and J. B. Grotberg, J. Appl. Physiol. 85, 333 (1998).

[12] F. F. Espinosa and R. D. Kamm, J. Appl. Physiol. 86, 391 (1999).

[13] K. Stephan et al., J. Micromech. Microeng. 17, N69 (2007).

[14] Y. Song et al., Med. Eng. Phys. (to be published).

[15] E. Delamarche et al., J. Am. Chem. Soc. 120, 500 (1998); J. M. Chen, C.-Y. Chen, and C.-H. Liu, Jpn. J. Appl. Phys. 47, 1683 (2008).

[16] M. M. Dias and A. C. Payatakes, J. Fluid Mech. 164, 305 (1986). 